Reprod. Nutr. Dévelop., 1981, 21 (5 B), 749-771.

\title{
Constituants alimentaires et motricité digestive
}

\author{
par Y. RUCKEBUSCH, L. BUENO, J. FIORAMONTI \\ Service de Physiologie ef Pharmacologie, I.N.R.A, \\ Ecole Nationale Vétérinaire, \\ 23, chemin des Capelles, 31076 Toulouse Cedex, France.,
}

\section{Summary. Dietary components and digestive motility.}

The motor profiles of the small and the large intestine are described in terms of migrating myoelectric patterns in the small bowel and of long to short spike burst ratios in the colon. The effects of such dietary components as carbohydrates, proteins and fats, and of the dietary fiber complex (including cellulose, hemicelluloses, lignin, pectins and gums) are examined in both dogs and pigs.

In carnivores, continuous, irregular duodeno-jejunal spiking activity, lasting 2 to $3 \mathrm{hrs}$ and masking the phases of quiescence and regular spiking in the pattern, is induced by both carbohydrates and proteins. In contrast, motility is inhibited for nearly $6 \mathrm{hrs}$ by an isocaloric lipid load, a component which, on the other hand, can stimulate concomitantly colonic activity. Similar but less important effects of a daily meal have been recorded in pigs. For example, the patterns were not disrupted but only delayed for 1 or $2 \mathrm{hrs}$. Among the dietary fiber complexes, cellulose, but not wheat bran and gums, increased jejunal transit time in dogs.

Dietary fiber, e.g. cereal fiber, considerably increases the number of long spike bursts in the canine colon, suggesting that the propulsive effect of this activity profile is stimulated by large amounts of colonic contents. In pigs on a milk diet, the profile of short spike burst activity reflects some degree of colon emptiness, and the addition of wheat bran as a bulking agent can halve transit time and double stool weight. Another way of adapting the gut to a high-residue diet is to increase the capacity of the caecocolic segment. No changes in motility have been recorded during such an adaptation in rabbits receiving, on an isocellulosic basis, beet pulp instead of alfalfa.

Finally, the level of spiking activity and its profiles in both the small and the large intestine are useful criteria in correlating motor changes and dietary components, as well as subsequent nutritional measures.

\section{Introduction.}

Les contractions de la paroi du tube digestif assurent le transit des aliments depuis la cavité buccale jusqu'à l'ampoule rectale. Au-delà de l'estomac, la motricité de l'intestin grêle assure, jusqu'à la valvule iléo-cæcale, la progression du chyme intestinal dont le volume est fonction de la quantité d'aliments ingérés ainsi que des sécrétions gastriques et duodéno-jéjunales. Au-delà de la jonction iléo-cæcale, le segment cæcum-côlon fonctionne comme un réservoir facilitant la déshydratation du contenu digestif résiduel et sa transformation en fèces. Le poids des fèces et leur teneur en eau 
sont fonction de l'importance et de la nature des résidus des cellules végétales après action des enzymes digestives. Des exemples extrêmes de cette situation sont apportés par les carnivores comme le chien, pour lequel la capacité digestive totale est de moins de 6 I pour $30 \mathrm{~kg}$, avec environ 26 p. 100 pour le secteur infestinal ; chez les omnivores comme le porc, l'intestin grêle et le segment cæco-colique occupent 71 p. 100 de la capacité digestive totale évaluée à 301 pour un porc de $70 \mathrm{~kg}$ (Kidder et Manners, 1978),

Le côlon représente 14 p. 100 de la capacité digestive chez le chien et 38 p. 100 chez le porc ; cela permet la consommation d'aliments contenant jusqu'à 15 p. 100 de cellulose ef l'élimination de $800 \mathrm{~g} / \mathrm{jour}$ de fèces chez les omnivores. Chez les carnivores, pour une ration contenant de 1 d̀ 4 p. 100 de cellulose, l'élimination fécale est inférieure à $100 \mathrm{~g} /$ jour.

La progression du contenu digestif au niveau de l'intestin grêle est assurée par des contractions coordonnées des couches, longitudinale et circulaire, de fibres musculaires. Les modalités de contraction sont assez univoques, en ce sens qu'une activité cyclique, représentée par la propagation depuis le pylore jusqu'à la valvule iléocæcale de contractions rythmiques, survient pendant 30 à 60 min toutes les 1,5 à $2 \mathrm{~h}$. Cette activité récurrente, décrite au début du siècle par Boldyreff chez le chien à jeun, a pour équivalent électromyographique le complexe myoélectrique (migrating myoelectric - or motor - complex : MMC) ; elle occupe comme l'activité mécanique, 30 à 50 p. 100 du temps d'enregistrement. La réponse motrice de l'intestin grêle aux variations de volume de son contenu ef à celles de la nature des constituants pourra donc être l'augmentation ou la réduction de la durée d'activité. L'autre modalité possible de la réponse est la répartition temporelle de l'intensité, de l'amplitude et de la fréquence des contractions, c'est-à-dire qu'elle concerne le type d'activité. Dans l'intervalle des périodes de repos de l'intestin grêle, l'alternance entre une phase assez longue (40$60 \mathrm{~min}$ ) où les contractions surviennent de façon irrégulière et une phase de 4 à 5 min durant laquelle les contractions sont maximales en amplitude et régulières en fréquence nous a fait distinguer dans chaque MMC, d'abord chez le rat (Ruckebusch et Fioramonti, 1975) puis chez le chien et le mouton (Bueno, Fioramonti et Ruckebusch, 1975), une phase d'activité irrégulière $(A I)$ et une phase d'activité régulière (AR). Cette terminologie, Al et $A R$, correspond aux équivalents électromyographiques des phases II et III du complexe, telles qu'elles ont été décrites à la même époque par Code et Marlett (1975) chez le chien.

Le côlon est ou bien un simple fube avec présence ou non d'haustrations; ou bien il est spécialisé dans le moulage des fèces aboutissant à la formation de crottes. Dans les deux cas, il présente des modalités de contraction également univoques, durant au moins 30 p. 100 du temps d'enregistrement. Un type d'activité se traduit par de brèves salves de potentiels, peu ou faiblement propagées : les variations de pression correspondantes sont nombreuses mais de faible amplitude (ondes de type I de Templeton et Lawson, 1931) ; elles suggèrent des modifications de tonus de la paroi. L'autre type d'activité correspond à de puissantes contractions (ondes de type II) propagées le long d'une partie du segment colique (Ruckebusch et Fioramonti, 1980a). A l'instar de l'intestin grêle, la réponse motrice du côlon pourra donc concerner la durée d'activité et la nature localisée ou propagée de cette activité. Dans le cas du jeune rat, soumis durant 3 à 5 mois à l'afflux massif mais intermittent de chyme gastrique (Ruckebusch ef Ferré, 1972), et du lapin en croissance recevant de façon continue un aliment riche 
en hémicelluloses (Candau, Bertrand et Fioramonti, 1978), la partie proximale de l'intestin ef le segment cæco-colique présentent moins des variations de la motriciłé qu'une adaptation morphologique du tractus digestif ; en d'autres termes, l'adaptation est davantage une augmentation de la capacité digestive que celle des phénomènes contractiles (voir Ruckebusch, 1981).

Dans l'analyse des interactions motricité-constituants alimentaires de la ration, nous distinguerons donc, à côté de l'adaptation morphologique, la fraction directement digérée par l'attaque enzymatique d'origine endogène (glucides, protides et lipides) et d'autre part les fibres alimentaires (Trowel, 1976) d'origine végétale, le plantix selon Spiller, Shipley et Blake (1978) - dont la fraction cellulosique est plus ou moins dégradée par la flore bactérienne du segment cæco-colique.

L'intérêt de cette voie d'approche est d'ordre physio-pathologique. La teneur en fibres alimentaires de la ration est en effet considérée comme un facteur déterminant de la durée du transit digestif, essentiellement celui du côlon chez l'homme et chez le porc (tabl. 1). L'apport quotidien de $50 \mathrm{~g}$ de son de blé contenant $40 \mathrm{p} .100$ de fibres et

TABLEAU 1

Relation entre la durée de transit des aliments ef la teneur en fibres alimentaires de la ration

\begin{tabular}{|c|c|c|c|c|}
\hline & $\begin{array}{l}\text { Teneur } \\
\text { en Fibres }\end{array}$ & $\begin{array}{c}\text { Poids } \\
\text { des Selles } \\
\text { (/jour) }\end{array}$ & Transit & Référence \\
\hline Homme : & & & Vitesse & \\
\hline - Milieu rural $\ldots \ldots \ldots \ldots \ldots \ldots \ldots$ & $\begin{array}{r}25 \text { p. } 100 \\
5 \text { p. } 100\end{array}$ & $\begin{array}{l}30-70 \mathrm{~g}(a) \\
20-25 \mathrm{~g}\end{array}$ & $\begin{array}{l}8-10 h(b) \\
20-30 h\end{array}$ & Walker, 1947 \\
\hline Homme : & & & Durée & \\
\hline $\begin{array}{l}\text { - Région rurale en Ouganda .... } \\
\text { - Militaires de la Royal Navy ... }\end{array}$ & $\begin{array}{l}+++ \\
+\end{array}$ & $\begin{array}{l}470 \mathrm{~g} \\
107 \mathrm{~g}\end{array}$ & $\begin{array}{l}36 \mathrm{~h} \\
69 \mathrm{~h}\end{array}$ & Burkitt, 1976 \\
\hline Porc : & & & Durée & \\
\hline $\begin{array}{l}\text { - Régime concentré } \ldots \ldots \ldots \ldots \ldots \ldots \\
\text { - Lait et son } \ldots \ldots \ldots \ldots \ldots \ldots \ldots\end{array}$ & $\begin{array}{r}15 \text { p. } 100 \\
8 \text { p. } 100\end{array}$ & $\begin{array}{l}808 \pm 63 \\
270 \pm 27\end{array}$ & $\begin{array}{l}40 h \\
66 h\end{array}$ & $\begin{array}{r}\text { Fioramonti et } \\
\text { Bueno, } 1980\end{array}$ \\
\hline
\end{tabular}

$\left({ }^{a}\right)$ Poids des matières sèches, $\left({ }^{b}\right)$ Temps d'apparition du colorant.

fixant quelque $300 \mathrm{~g}$ d'eau est capable de doubler le poids des selles (Cummings et al., 1976), ce en quoi il remplace avantageusement les laxatifs dans la constipation chronique (Frexinos, 1979).

\section{Activité propulsive de l'intestin et du côlon.}

\subsection{Profil moteur de l'intestin}

Intestin grêle. - Il esł désormais bien établi que la propulsion du contenu de la lumière infestinale n'est pas régulière ; elle est intermittente, même dans le cas où l'évacuation gastrique est continue comme chez les herbivores polygastriques. En effet, la progression de la phase liquide tributaire de l'activité motrice de l'intestin est maxi- 
male durant les 10 à 15 dernières minutes d'une phase d'activité irrégulière, de sorte que l'écoulement le plus abondant du contenu digestif à travers une canule chez un animal fisłulé survient juste avant le développement de la phase d'activité régulière (fig. 1). Les contractions circonférentielles réitérées et propagées sur une courte dis-

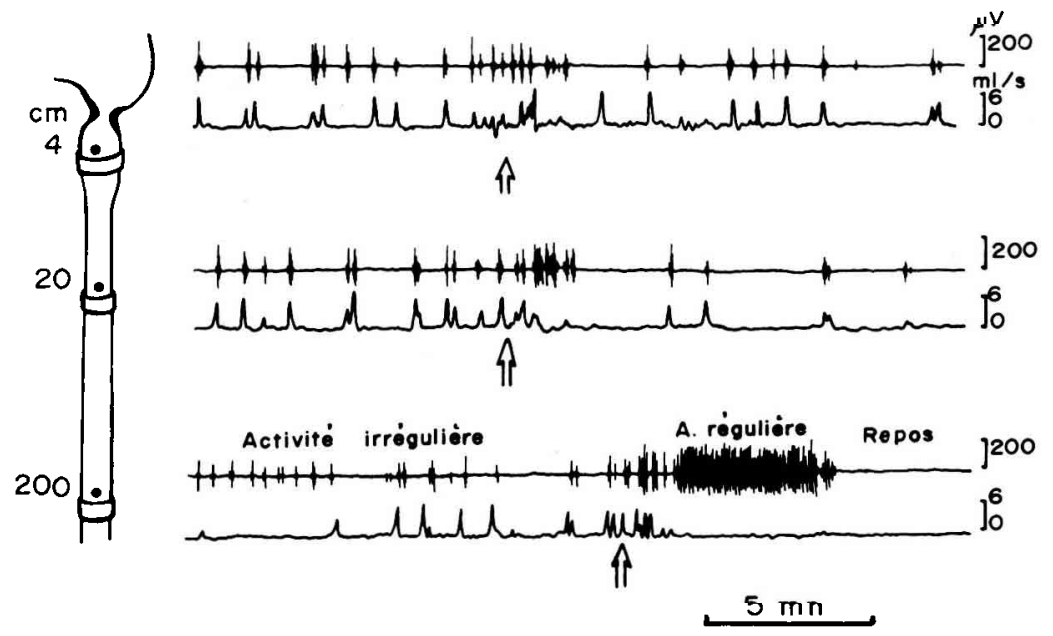

FIG. 1. - Mécanisme de la propulsion liquide. L'effet propulsif des contractions de la paroi intestinale est maximal en avant de la zone de haute pression créée par la phase d'activité régulière du complexe.

tance de la phase d'activité régulière créent une zone de haute pression qui pousse devant elle le contenu digestif au fur et à mesure de sa lente migration (Bueno, Fioramonti et Ruckebusch, 1975). Le gradient de pression ainsi créé interdit tout reflux liquide vers l'amont et favorise l'efficacité propulsive des contractions péristaltiques (activité irrégulière) qui prennent naissance en aval (voir Weisbrodt, 1981).

La riposte motrice de l'intestin grêle à une augmentation du niveau global des ingesta varie selon les espèces : la tendance générale est l'augmentation de la durée d'activité. Chez le chien, la durée des phases d'Al est négativement corrélée avec le temps écoulé depuis le dernier repas. Immédiatement après le repas, l'augmentation considérable du débit pylorique s'accompagne d'une activité motrice continue qui persitera de 8 à 11 h selon la quantité d'aliments ingérés. Cette disparition des périodes de repos encore appelée "désorganisation postprandiale » ou "disruption des $M M C$ » s'observe pour un débit duodénal supérieur à $120 \mathrm{ml} / \mathrm{h}$ chez le chien et $300 \mathrm{ml} / \mathrm{h} \mathrm{chez}$ le porc (Buenc et Ruckebusch, 1978). Chez le mouton, lorsque le niveau d'ingesta est élevé par suralimentation, la durée d'activité motrice est accrue, comme chez le chien par un allongement des phases d'activité irrégulière ; une autre modification caractéristique chez le mouton est la présence de complexes surnuméraires migrant le long du jéjunum (Bueno, 1977). Il est à noter que le terme « disruption » consacré par l'usage peut prêter à confusion car le profil moteur caractérisé par la disparition des MMC s'observe aussi dans certaines conditions pathologiques (Laplace, 1980).

Sur le plan fonctionnel, l'activité motrice continue postprandiale de l'intestin grêle assure un débit intestinal élevé et une viłesse de transit en permanence proche 
de celle observée de façon transitoire chez l'animal à jeun. Durant la période d'activité motrice continue, un marqueur de la phase liquide se déplace à une vitesse qui est proche de celle observée en aval de la zone de haute pression développée par la phase d'activité régulière d'un complexe myoélectrique (fig. 2).

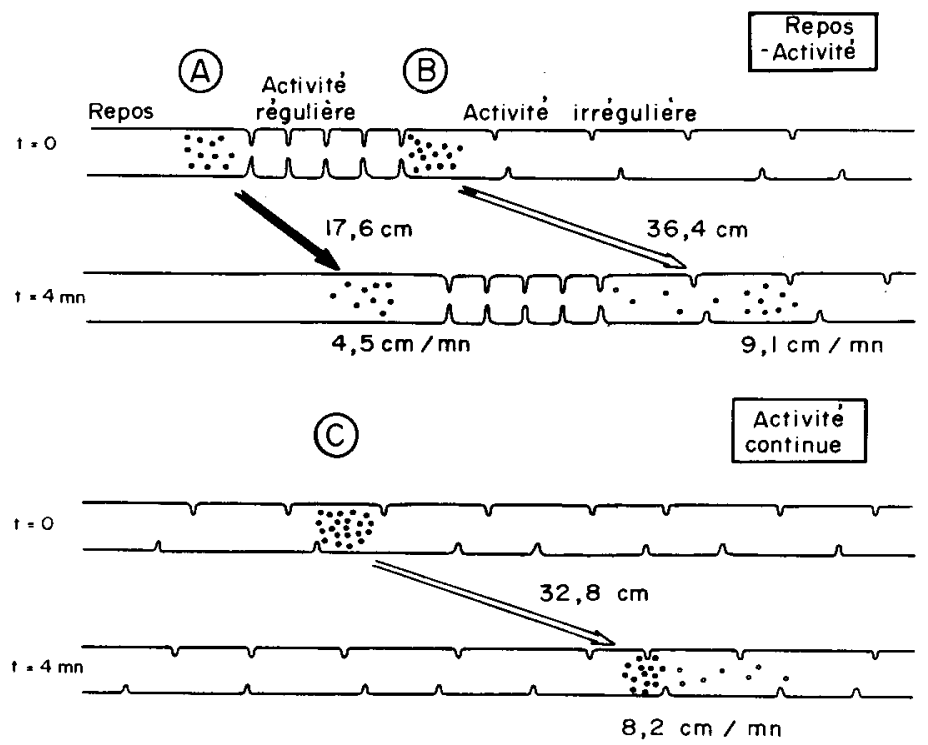

FIG. 2. - Alternance activité-repos et activité continue postprandiale. La distance parcourue en $4 \mathrm{~min}$ chez le rat par un colorant injecté avant la zone de pression créée au niveau jéjunal par la phase d'activité régulière est de $36 \mathrm{~cm}(A)$ au lieu de $18 \mathrm{~cm}$ pour une injection faite durant la phase de repos (B). La distance parcourue pour une activité continue de l'intestin est de $33 \mathrm{~cm}$ (C).

Gros intestin. - Le profil moteur du côlon humain est caractérisé sur le plan radiologique par deux sortes de contractions. Les unes localisées et stationnaires, assurent la segmentation du contenu colique qui reste pratiquement sur place pendant plusieurs heures, en particulier au niveau du côlon proximal. Les autres, constituées par des anneaux de constriction précédés d'une zone de relâchement, se propagent à une vitesse de l'ordre de $2 \mathrm{~cm} / \mathrm{s}$ et sont responsables du transport oral et aboral du contenu colique. Le transfert du contenu vers la partie distale du côlon résulte d'une prépondérance des contractions propagées vers l'aval par rapport à celles propagées vers l'amont. Ce phénomène d'antipéristaltisme décrit chez le lapin au début du siècle par Elliott ef Barclay-Smith (1904) est surtout net pour la partie proximale du côlon.

L'activité localisée du côlon se traduit sur le plan électromyographique par des salves de potentiels brèves (short spike bursts : SSB) d'une durée inférieure à $5 \mathrm{~s}$; elles correspondent, en particulier dans le cas de série à fréquence élevée (10 à $15 / \mathrm{min}$ ), à une activité tonique de la paroi. L'activité propagée comprend de longues salves de potentiels (long spike bursts : LSB) d'une durée supérieure à 15 s propagées dans les sens iso- et antipéristaltique sur plusieurs centimètres : elles correspondent à une activité phasique (fig. 3). Les preuves directes du rôle fonctionnel des deux types de 

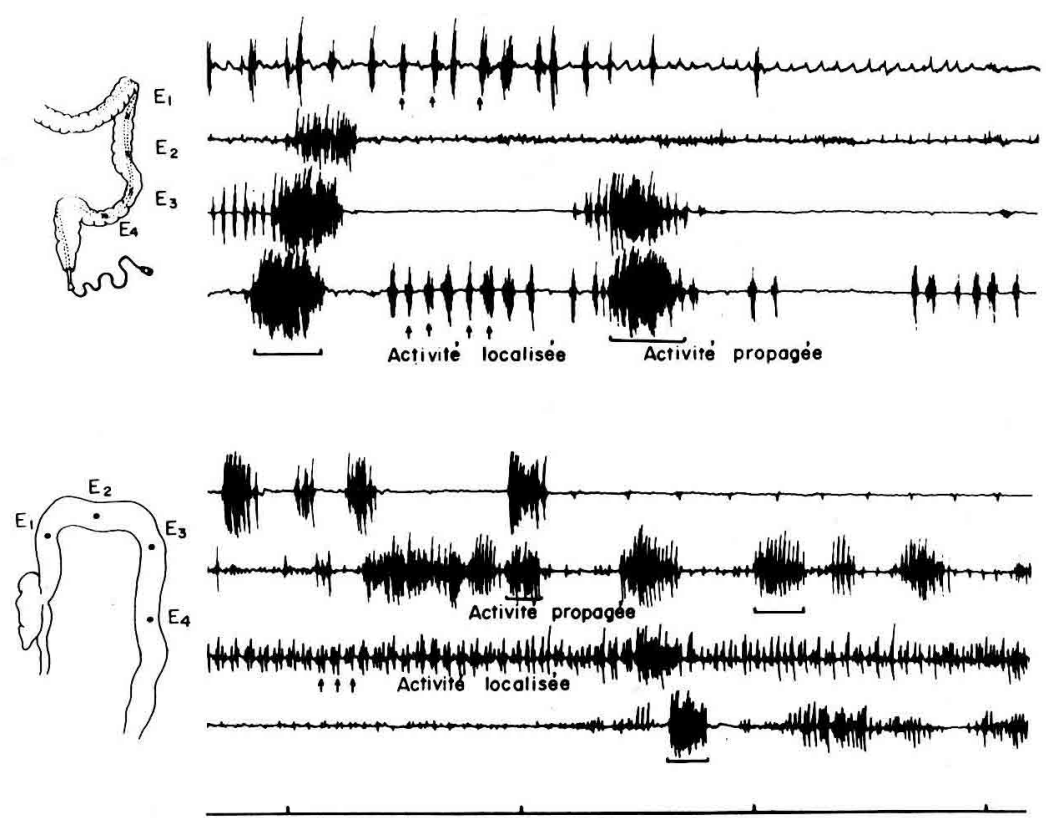

\section{Minutes}

FIG. 3. - Activité localisée et propagée du côlon chez l'homme ef le chien. Les salves de potentiel propagées ont une durée de $20 \mathrm{~s}$ chez l'homme ef de $7 \mathrm{~s}$ (côlon ascendant) à $30 \mathrm{~s}$ (côlon descendant) chez le chien. L'activité localisée est dans les deux cas représentée par des salves de 1 à 3 s dont la fréquence de répétition est élevée.

TABLEAU 2

Profil moteur du côlon descendant chez l'homme sain ou colopathe et chez le chien avant ef après colostomie

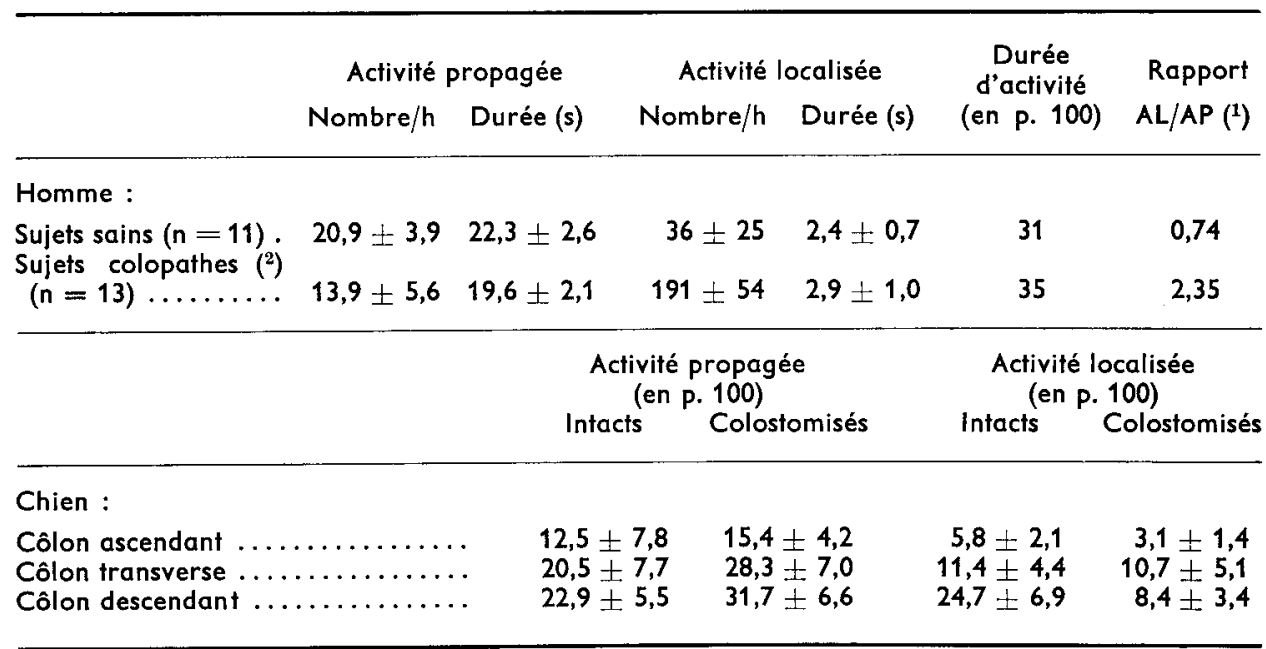

(1) Rapport activité localisée/activité propagée. $\left({ }^{2}\right)$ En majorité constipation. 
contractions coliques sont l'existence d'une activité localisée fortement accrue chez les colopathes atteints de constipation (Bueno ef al., 1980) ef la disparition de ce type d'activité localisée au niveau du côlon distal chez le chien colostomisé. La création d'un anus artificiel en avant de l'ampoule rectale supprime en effet le frein pelvien (tabl. 2).

La physiologie comparée apporte d'autres arguments dans ce sens. Les espèces dont les fèces sont simplement moulées (chien ou porc) présentent les deux types d'activité à tous les niveaux du côlon. L'activité localisée est prédominante en zone distale et l'activité propagée iso- et antipéristaltique est particulièrement nette en zone proximale. Les espèces qui éliminent leurs fèces sous la forme de crottes (lapin, mouton) disposent de segments coliques spécialisés ; le côlon proximal chez le lapin et le côlon spiral chez le mouton sont le siège d'une activité localisée quasi permanente à laquelle s'ajoute de temps à autre une activité propagée en direction aborale. Un mécanisme où quelques contractions iso-péristaltiques se surimposent à une activité tonique permanente peut expliquer la forme et la dureté des fèces. Chez le lapin, la production de cæcotrophes ou fèces molles résulte ainsi d'une réduction de 80 p. 100 de l'activité localisée du côlon proximal (Fioramonłi ef Ruckebusch, 1976). Ce phénomène se retrouve dans les troubles diarrhéiques non spécifiques et dans la production de bouses chez la vache, dont l'anatomie du gros intestin est pourtant analogue à celle du mouton. La motricité se différencie sur le plan fonctionnel par une faible activité localisée du côlon spiral chez les bovins par rapport aux ovins (Ruckebusch et Fioramonti, 1980b). La lente progression 8 à 10 fois par jour de phases d'hyperactivité tout le long du côlon spiral et du côlon terminal chez la vache rappelle les 7 à 11 contractions propagées postprandiales observées chez l'homme. Enfin, la formation de croties ovoïdales et non sphériques chez le rat semble relever également d'une forte activité localisée, mais à laquelle s'ajoute une activité propagée dans le sens iso- et antipéristaltique (Fioramonti ef al., 1980).

TABLEAU 3

Temps de transit et débit intestinal chez le chien ef le mouton

\begin{tabular}{|c|c|c|c|}
\hline & $\begin{array}{l}\text { Temps de } \\
\text { transit } \\
(\mathrm{min} / \mathrm{m})\end{array}$ & $\begin{array}{l}\text { Vitesse de } \\
\text { propagation } \\
\text { (cm/min) }\end{array}$ & $\begin{array}{c}\text { Débit jéjunal } \\
(\mathrm{m} / / \mathrm{h})\end{array}$ \\
\hline \multicolumn{4}{|l|}{ Chien : } \\
\hline 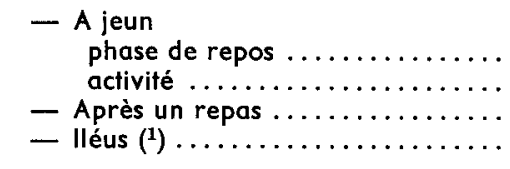 & $\begin{array}{r}23,3 \pm 4,1 \\
6,2 \pm 1,3 \\
4,3 \pm 1,6 \\
46,1 \pm 16,2\end{array}$ & $\begin{array}{r}4,3 \pm 2,6 \\
16,1 \pm 3,4 \\
23,2 \pm 8,6 \\
2,2 \pm 0,5\end{array}$ & $\begin{array}{r}104 \pm 26 \\
333 \pm 78 \\
52 \pm 21\end{array}$ \\
\hline \multicolumn{4}{|l|}{ Mouton : } \\
\hline $\begin{array}{l}\text { - Repos } \ldots \ldots \ldots \ldots \ldots \ldots \ldots \ldots \ldots \ldots \\
- \text { Activité } \ldots \ldots \ldots \ldots \ldots \ldots \ldots \ldots \ldots \ldots \\
- \text { Iléus }\left({ }^{1}\right) \ldots \ldots \ldots \ldots \ldots \ldots \ldots\end{array}$ & $\begin{array}{r}18,8 \pm 5,8 \\
5,7 \pm \quad 1,6 \\
72,5 \pm 5,2\end{array}$ & $\begin{array}{r}5,3 \pm 1,6 \\
17,5 \pm 4,1 \\
1,3 \pm 0,3\end{array}$ & $\begin{array}{r}380 \pm 72 \\
20 \pm 22\end{array}$ \\
\hline
\end{tabular}

(1) Obtenu au cours de l'injection intrapéritonéale de sérum salé hypertonique (Bueno, Fioramonti et Ruckebusch, 1975). 


\subsection{Particularités du transit digestif.}

Carnivores. - L'activité motrice continue du duodéno-jéjunum du chien assure à la suite d'un repas le transfert de plus de $300 \mathrm{ml} / \mathrm{h}$ de contenu digestif. L'activité devient discontinue 6 à $10 \mathrm{~h}$ plus tard, lorsque le débit intestinal horaire n'excède plus $120 \mathrm{ml}$ et la vitesse de transit de l'ordre de $23 \mathrm{~cm} / \mathrm{min}$ diminue fortement pendant les périodes de repos : 4 à $5 \mathrm{~cm} / \mathrm{min}$ (tabl. 3). Une activité motrice minimale ef un débit n'excédant pas quelque $50 \mathrm{ml} / \mathrm{h}$ s'observent dans le cas où la totalité de l'intestin grêle est au repos, comme dans le cas de l'iléus postopératoire ; la vitesse de transit est alors inférieure à $2 \mathrm{~cm} / \mathrm{min}$ (Bueno, Fioramonti ef Ruckebusch, 1975). Entre les valeurs extrêmes de débit postprandial et le débit limité aux sécrétions comme dans le cas d'une alimentation parentérale, la durée des phases d'activité irrégulière au niveau jéjunal reflète l'importance de la qualité de contenu digestif propulsée vers le côlon (fig. 4).
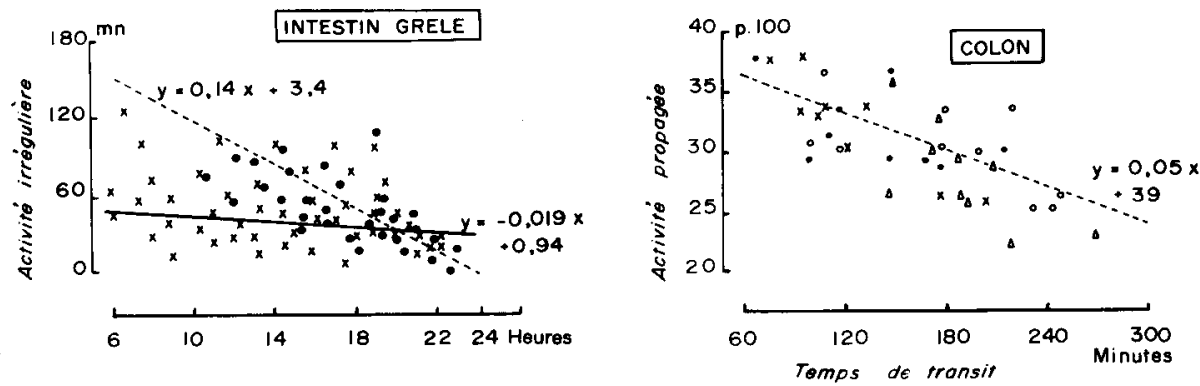

FIG. 4. - Temps de transit intestinal chez le chien. L'activité motrice de l'intestin exprimée par la durée des phases d'activité irrégulière est d'autant plus faible que l'on s'éloigne de l'heure du repas (•). Au bout de $24 \mathrm{~h}$, elle est proche de celle qui persiste dans le cas d'une alimentation parentérale $(X)$. Au niveau du côlon le pourcentage d'activité propagée mesurée chez 4 chiens est directement corrélée au temps mis par un marqueur pour se déplacer du côlon proximal jusqu'en zone distale.

Pour le gros intestin des carnivores, le rapport activité propagée/activité localisée est également un facteur déterminant du transit digestif. Ainsi, on trouve une relation directe entre le pourcentage du temps d'enregistrement d'activité propagée et le temps de transit qui varie spontanément de 60 à 280 min pour le côlon (fig. 4) ; il est impératif cependant pour mettre en évidence une telle relation d'éviter toute gêne à l'évacuation du contenu colique par le sphincter anal.

L'épaisse musculature de l'intestin grêle du chien explique sans doute aussi ses faibles facultés d'adaptation à un débit pylorique accru. Chez le mouton, la paroi intestinale est mince et le degré moyen de réplétion bequcoup plus élevé ; or, une activité motrice comparable à celle du chien et occupant 50 p. 100 du temps d'enregistrement assure un transit environ trois fois supérieur en volume (380 au lieu de $104 \mathrm{ml} / \mathrm{h}$ ).

Préruminants. - Avant le sevrage, la motricité digestive du veau ou de l'agneau est comparable dans son ensemble à celle d'un monogastrique adulte. Le lactose, environ 38 p. 100 de la matière sèche du lait de vache, est hydrolysé dans le premier tiers de l'intestin grêle.

A l'exception de la phase d'activité continue consécutive à la tétée, les périodes d'activité motrice de l'intestin grêle se succèdent selon une périodicité de l'ordre de 
55 min. Le schéma imaginé par Dardillat (1977) rend compte du parallélisme entre la durée du transit d'un marqueur inerte et la migration lente de la phase d'activité régulière (fig. 5). Les contractions duodénales propulsent des bols de 5 à $10 \mathrm{ml}$ dont le

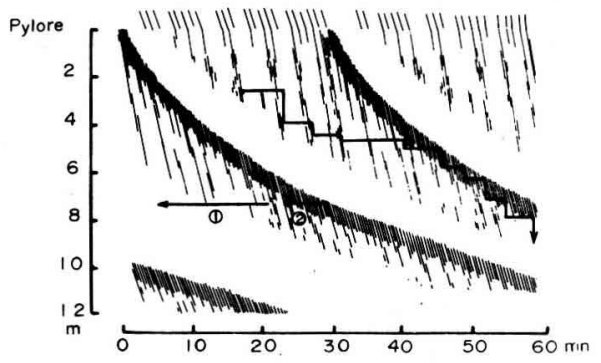

FIG. 5. - Migration des complexes myoélectriques chez le veau. Chaque tiret représente dans ce schéma la propagation d'une salve de potentiels sur l'antre et l'intestin grêle. Le quart seulement des salves est représenté pour plus de clarté. Le trajet suivi par une particule inerte depuis le duodénum jusqu'à l'iléum est indiqué ; il est parallèle à celui de la phase d'activité régulière et en relation directe avec la migration de cette dernière en zone distale. Les tirets correspondent à l'activité irrégulière (1) et à la migration de la phase d'activité régulière (2) (d'après Dardillat, 1977).

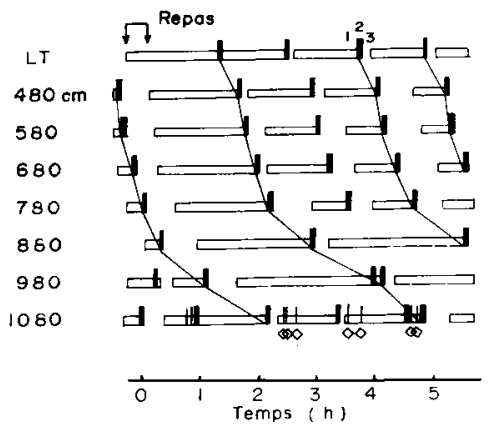

FIG. 6. - Propagation postprandiale des complexes myoélectriques chez le porcelet. L'ingestion d'un repas de $660 \mathrm{~g}$ chez un porcelet de $26 \mathrm{~kg}$ ne fait que prolonger l'intervalle séparant la propagation de deux complexes myoélectriques. L'activité électrique en zone iléale ef distale présente de brèves périodes d'activité maximale $(\diamond)$. Elles correspondent à des passages du contenu iléal dans le côlon (d'après Rayner ef al., 1980).

contenu se dépose le long de la paroi sur des distances de 0,5 à $1 \mathrm{~m}$. En dehors du passage de ces ondes contractiles et au-delà de leur évanescence, l'intestin paraît vide de sorte que l'activité régulière joue le rôle essentiel et permanent de ramener et de rassembler dans la zone de propulsion le contenu digestif étalé le long de la muqueuse (Dardillat et Marrero, 1977).

L'intérêt majeur du modèle préruminant est une extrême sensibilité digestive. La moindre atteinte se traduit le plus souvent par une parésie gastro-duodénale accompagnée de diarrhée. Dans ces conditions, le profil moteur est totalement désorganisé et le débit intestinal ne paraît plus lié aux salves propulsives. Celles-ci sont propagées sur de 
faibles distances et leur fréquence n'est pas accrue durant les $6 \mathrm{~h}$ consécutives à un repas. Bien que l'état de réplétion digestive soit élevé, le nombre total des contractions n'est pas augmenté : les contractions antrales chez un veau atteint de diarrhée n'excèdent pas 390 (au lieu de 670) et celui des contractions duodéno-jéjunales 19 (au lieu de 67).

Omnivores. - Le porc est le modèle d'étude par excellence de l'influence des constituants alimentaires et des modalités de leur distribution. En effet, contrairement au chien, il accepte de fractionner sa ration en de multiples repas et d'autre part l'estomac joue un rôle tampon appréciable puisqu'il ne se vide jamais complètement dans l'intervalle des repas (Rérat et Lougnon, 1963). Enfin, la teneur en matière sèche du contenu cæco-colique s'élève progressivement de 13 (cæcum) à 25 p. 100 (côlon distal), comme chez le mouton (Hecker ef Grovum, 1975). Elle est susceptible d'atteindre 37 p. 100 lorsque le lait est le seul aliment disponible ; et dans ces conditions, la durée moyenne du transit peut atteindre $120 \mathrm{~h}$ (Canguilhem et Labie, 1977).

Bien que dans les 15 min qui suivent un repas, près de 40 p. 100 du contenu gastrique franchisse le pylore sous un volume égal (compte tenu des sécrétions salivaire et gastrique) à celui de la totalité du repas consommé (Auffray, Martinet et Rérat, 1967), le porc conserve volontiers l'alternance repos-activité motrice de l'intestin grêle. Lorsqu'il reçoit sa ration en plusieurs repas, le porc ne modifie même pas la distribution des 18 périodes quotidiennes d'activité (Ruckebusch et Bueno, 1976). Le seul effet de l'ingestion d'un repas standard est un allongement considérable de la phase d'activité irrégulière (104 au lieu de $77 \mathrm{~min}$ ). L'étude cinéradiographique concomitante montre que la migration des phases d'activité régulière fractionne le contenu radio-opaque de la totalité de l'intestin grêle en trois ou quatre segments qui se déplacent à la même vitesse (fig. 6). Ils mettent environ $190 \mathrm{~min}$ pour parcourir les $10 \mathrm{~m}$ d'intestin qui séparent le ligament de Treitz de la valvule iléo-cæcale chez un porc de $30 \mathrm{~kg}$ en phase digestive au lieu de $210 \mathrm{~min}$ durant les périodes interprandiales (Rayner ef al., 1980).

Enfin, la concentration colique en acides gras volatils (acides acétique, propionique et butyrique) issus des fermentations microbiennes est environ trois fois plus élevée chez le porc que chez le chien. L'apport énergétique représenté par les acides gras volatils issus des fermentations cellulosiques d'un régime contenant 9,2 p. 100 de cellulose est de 11 p. 100 environ ; cet apport n'est plus que de 5 d̀ 7 p. 100 selon le poids de l'animal (48 et $89 \mathrm{~kg}$ ) pour un régime contenant 7,5 p. 100 de cellulose : l'apport le plus faible s'observe chez les sujets les plus lourds dont le transit est également le plus rapide (Kass, Van Soest et Pond, 1980).

\section{Influence de l'absorption sur le profil moteur.}

\subsection{Influences excito-motrices.}

Glucides. - Dès 1975, nous avons montré chez le chien à jeun depuis $18 \mathrm{~h}$ que l'administration orale de glucose déclenche une activité motrice continue de la zone duodéno-jéjunale, activité analogue à celle qui suit un repas et associée à une augmentation de l'insulinémie (Bueno et Ruckebusch, 1976). Une action directe sur des chémorécepteurs locaux est probable puisque l'injection par la veine porte ou la veine jugulaire de quantités équivalentes de glucose n'augmente la motricité que de façon 
très passagère chez le chien (fig. 7). Enfin, la durée de l'activité continue postprandiale est augmentée de 25 p. 100 en remplaçant environ 8 p. 100 des protéines de la ration par une quantité équivalente de glucides.

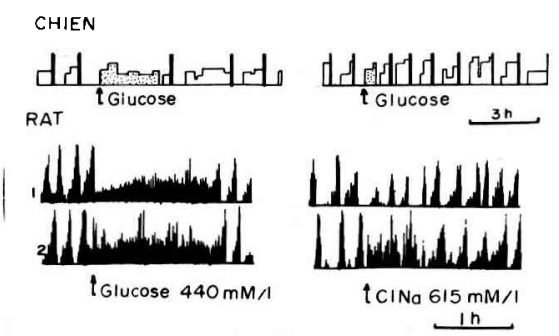

FIG. 7. - Effet excito-moteur spécifique du glucose par voie orale sur l'intestin grêle chez le chien ef le rot. Le glucose par voie orale produit chez le chien une activité motrice jéjunale continue durant $3 \mathrm{~h}$ pour une dose de $1 \mathrm{~g} / \mathrm{kg}$. L'effet esł passager pour la même dose par voie endoveineuse. La perfusion intraduodénale de glucose $(5 \mathrm{ml}$, à 8 p. 100) exacerbe l'activité duodéno-jéjunale recueillie en deux points ( 1 et 2 ) distants de $10 \mathrm{~cm}$ chez le rat, alors qu'une solution hypertonique de $\mathrm{CINa}$ $(10 \mathrm{ml}$ à 3,6 p. 100) est sans effet.

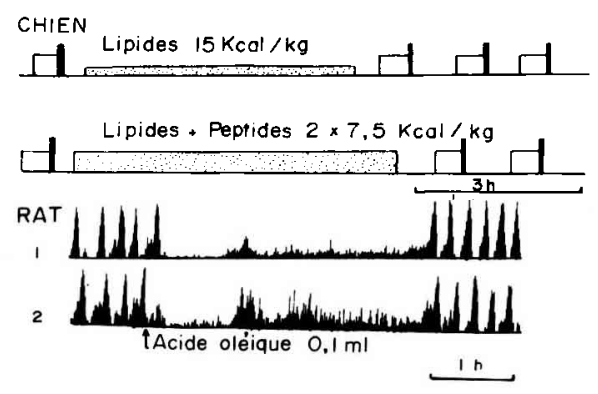

FIG. 8. - Effets d'une même charge colorique de lipides et protides sur la motricité jéjunale. Les périodes de repos disparaissent chez le chien recevant un mélange de triglycérides et de peptides connus à la suite d'un repas. En réalité, cet effet résulte d'une inhibition motrice due aux lipides, masquée dans le cas de leur association à des protides. Chez le rat, l'inhibition motrice due à l'acide oléique dure environ $3 \mathrm{~h}$ au niveau duodéno-jujénal et plus de $10 \mathrm{~h}$ au niveau antro-pylorique.

Le rat répond de manière analogue à la perfusion duodénale de glucides et une action spécifique est probable car il n'est pas possible de reproduire l'action excitomotrice par des solutions salines hyperosmotiques (fig. 7).

Le porc recevant du lactose dont le taux d'absorption n'est que de 15 p. 100 en $8 \mathrm{~h}$ chez l'adulte au lieu d'amidon de maīs ne présente aucune hyperactivité postprandiale retard. De surcroît, l'activité motrice jéjuno-iléale est faible et disparaît ; cette hypomotilité qui va de pair avec une forte augmentation (35,7 p. 100) du volume des digesta est plutôt liée à un appel d'eau par effet osmotique qu'à une intolérance digestive au lactose, non dégradé par insuffisance de lactase endogène (Laplace, 1978).

Protides. - L'administration intraduodénale d'un mélange d'amino-acides équivalent à $450 \mathrm{mM} / \mathrm{l}$ possède, chez le rat, des effets exciło-moteurs proches de ceux observés avec les glucides (Ruckebusch ef Fioramonti, 1975). Un contrôle hormonal esł possible puisque seuls, par voie intraveineuse, les amino-acides insulino-sécréteurs, la 
L-leucine et la L-arginine $(0,2 \mathrm{~g} / \mathrm{kg})$, possèdent la propriété d'accroître l'activité motrice duodéno-jujénale chez le chien (Ruckebusch et Bueno, 1977). Néanmoins, l'association de glucides aux constituants protidiques d'un repas n'entraîne pas pour autant un cumul des effets sur la motricité : l'effet global est inférieur à celui obtenu pour les seuls constituants glucidiques.

Un point discuté reste le rôle de l'absorption dans la mise en jeu de la réponse motrice. A la « dose isocalorique » de $30 \mathrm{kcal} / \mathrm{kg}$, la durée des effets de la lactalbumine est environ deux fois moins longue ( $2 \mathrm{~h}$ au lieu de $3,5 \mathrm{~h}$ ) que celle du lactose (de Wever ef al., 1978) ; aucune des nombreuses interprétations possibles n'est décisive en l'absence du contrôle de l'absorption.

\subsection{Influence inhibitrice des lipides.}

Le retard de l'évacuation gastrique d'un repas riche en graisses a suscité de nombreux travaux en relation avec la motricité antrale et/ou duodénale. L'acide oléique et non l'huile de paraffine inhibe fortement chez le rat la motricité duodéno-jéjunale et celle de l'estomac, avec absence durant près de $3 \mathrm{~h}$ de complexes myoélectriques. Cet effet a été comparé à tort à l'absence de complexes après un repas riche en glucides. En effet, dans le cas des lipides, il s'agit d'une hypomotilité analogue à celle de l'iléus paralytique et non d'une activité motrice continue. L'hypomotilité persiste environ $6 \mathrm{~h}$ chez le chien pour un repas d'épreuve d'huile d'arachide apportant $30 \mathrm{kcal} / \mathrm{kg}$ (de Wever et al., 1978). Le retard de la vidange gastrique et l'inhibition de la motricité antrale en cause dans les conditions naturelles sont exclus dans le cas où les lipides sont introduits dans une anse intestinale isolée (Schang et al., 1978) ; dans ce cas, l'inhibition apparaît sur la totalité de l'intestin grêle. Comme pour l'association glucides-protides, l'association lipides-protides aboutit à une neutralisation de l'effet inhibiteur spécifique des matières grasses sur la motricité duodéno-jéjunale (Schang et al., 1980) (fig. 8).

De telles interactions s'observent aussi chez le veau. Dans les conditions de l'élevage industriel, une partie des lipides est remplacée par des matières amylacées ; la présence d'une quantité importante d'amidon en substitution d'une partie des matières grasses diminue la teneur en matière sèche des fèces (Thivend, 1979). Le pouvoir diarrhéique de l'ensemble des glucides de l'aliment (lactose, glucose, dextrines et amidon cru) peut venir de ce que les capacités digestives des enzymes de l'hôte sont dépassées. La présence de glucides simples non digérés dans l'intesfin grêle puis le gros intestin peut également créer un appel d'eau par osmose dans la lumière intestinale. En outre, il est probable que l'absence de lipides favorise l'évacuation gastrique et limite ainsi le temps de séjour dans la caillette.

Au niveau intestinal, la substitution d'une partie des matières grasses de l'aliment par des quantités importantes de produits amylacés favorise une activité de type continue, propulsive ; elle réduit le temps de transit, ce qui explique l'augmentation de l'hydratation des fèces.

Chez le porc, la digestion des lipides semble extrêmement efficace en raison d'une forte activité lipasique et d'une abondante sécrétion biliaire ainsi que de leurs adaptations à la nature des aliments (Corring et al., 1979). La puissance digestive de l'intestin grêle du porc se retrouve également pour les glucides puisque le pourcentage de digestion des hémicelluloses y atteint 38 p. 100 (Kass, Van Soest et Pond, 1980). Enfin, la 
fraction non digérée de l'amidon qui atteint le segment cæco-colique pour y subir des fermentations microbiennes avec production d'acides gras volatils est relativement faible (Rérat, 1978).

\subsection{Réponses cæco-coliques.}

L'augmentation de l'activité motrice du cæcum et du côlon au cours de l'ingestion d'un repas avait été observée chez le chat dès 1902. Puis furent décrites, pour ce réflexe gastro-colique, des origines psychiques, buccopharyngées, gastriques et même duodénales. Les modifications observées vont de la simple élévation globale de la pression endoluminale du côlon à l'apparition d'une intense activité propulsive. Les expériences de section et de stimulation vagale et sympathique sont en faveur d'un contrôle purement nerveux de ce réflexe (Holdstock et Misiewicz, 1970 ; Snape et a l., 1979). Enfin, il a été établi que la nature de la réponse évoquée était fonction du degré de réplétion du côlon. Le réflexe est localisé et sous la dépendance de mécanismes cholinergiques et adrénergiques dans le cas où le côlon est vide ; la réponse est propulsive et le mécanisme seulement cholinergique dans le cas où le côlon est plein (Tansy et Kendall, 1973).

Une augmentation de la motricité colique également liée à l'ingestion d'un repas apparaît plus tardivement lorsque le contenu digestif franchit la valcule iléo-cæcale. Cette réponse est évidemment le propre des espèces dont la quantité d'aliment consommée spontanément en seul repas est suffisamment importante. Chez le chien et le porc recevant un repas par jour, la réponse postprandiale est toujours biphasique. La vue et l'ingestion de l'aliment déclenchent une activité contractile propagée pendant 5 à 10 min sur le seul côlon proximal chez le chien et sur le côlon proximal et hélicoïdal chez le porc. Après une période d'inhibition motrice (chien) ou de moindre activité (porc) de 60 à $90 \mathrm{~min}$, une deuxième phase d'hyperactivité de type propagée correspond à l'arrivée du contenu iléal dans le côlon. Ultérieurement, des phases d'hyperactivité colique coïncideront avec l'arrivée des complexes myoélectriques en zone iléale.

Le mécanisme par lequel les constituants alimentaires évoquent cette réponse tardive, également observée chez l'homme (Kirwan et Smith, 1976), est mal connu. Une action directe du contenu digestif est peu probable à en juger par l'absence de modifications de la motricité du gros intestin liée à l'ingestion alimentaire chez le mouton (Fioramonti et Ruckebusch, 1979) et par son apparition lorsque la ration quotidienne est consommée en une période unique de $4 \mathrm{~h}$. La fréquence des contractions du cæcum et du côlon proximal s'élève alors, moins de 15 min après le début du repas, de $25 \mathrm{p}$. 100 environ ef pendant 4 à $5 \mathrm{~h}$. Or, cette hyperactivité cæco-colique ne peut être liée à une éventuelle augmentation du débit iléal ; celui-ci ne varie pratiquement pas (Goodall et Kay, 1965).

Un apport calorique minimal du repas est-il nécessaire pour déclencher la réponse motrice précoce et tardive du côlon au repos? Les effets de repas qui ne diffèrent que par cet aspect sont en faveur d'une telle origine (Snape, Matarazzo et Cohen, 1978) ; malheureusement, il n'a pas été tenu compte de leur teneur en matière sèche de sorte que l'incidence du facteur calorique sur la motricité colique ne peuł être tenue pour démontrée. En revanche, l'ingestion de matières grasses favorise, tout au moins chez l'homme, la réponse immédiate et stimule pendant une heure environ la motricité du côlon. Les acides aminés ne possèdent par eux-mêmes aucun effet, mais s'opposent à 
celui des lipides (Wright et al., 1980). Chez le porc, le lactose non digéré dans l'intestin grêle accroît la motricité du cæcum lorsqu'il y pénètre; dans ce cas précis, l'influence de l'acide lactique est possible (Laplace, 1978).

L'intervention de facteurs hormonaux est souvent soupçonnée depuis les observations de Connell et Logan (1967) relatives à l'effet excito-moteur de la gastrine sur le côlon proximal.

\section{Interactions motricifé-constituants « indigestibles ».}

\subsection{Effet «fibres alimentaires ».}

Définition. - Les «fibres alimentaires», définies comme les substances d'origine végétale de la ration non digérées, correspondent pratiquement au « résidu indigestible » chez un carnivore; en revanche, une fraction importante est dégradée par les micro-organismes du tractus digestif en acides gras volatils chez un herbivore. Les omnivores occupent une position intermédiaire pour laquelle les fibres ont un triple rôle ; celui de ballast avec ses conséquences sur le temps de transit intestinal (effet fibres), l'utilisation des produits de dégradation (aspects métaboliques) ainsi qu'une action pharmacodynamique des métabolites sur la paroi, en particulier sa vascularisation.

Les fibres sont des polymères et, d'une façon générale, des polysaccharides, à l'exception de la lignine (Rinaudo, 1980). La cellulose est un polymère du $\beta$ 1-4.Dglucose de 3000 à 10000 unités sans ramifications. Les hémicelluloses, c'est-à-dire la fraction végétale soluble en milieu alcalin dilué, groupent une grande variété de polymères généralement ramifiés contenant 150 à 250 unités de 2 à 4 oses; environ 250 variétés d'hémicelluloses sont actuellement connues et le terme de pentosane est parfois utilisé pour les désigner en raison de leur forte teneur en pentoses.

Les pectines sont des polymères de l'acide $\beta$ 1-4.D-galacturonique fortement substitués alors que les gommes et mucilages sont des polymères hautement ramifiés des acides galacturonique et glucuronique.

TABLEAU 4

Effet des fibres alimentaires sur la motricité intestinale chez le chien

\begin{tabular}{|c|c|c|c|c|}
\hline & \multirow{2}{*}{$\begin{array}{l}\text { Témoin } \\
500 \mathrm{~g} \\
\text { d'aliment }\end{array}$} & \multicolumn{3}{|c|}{ Addition de $50 \mathrm{~g}$ de } \\
\hline & & Son & Cellulose * & Gomme** \\
\hline $\begin{array}{l}\text { Désorganisation } \\
\text { posfprandiale }\end{array}$ & Durée (h) & \multicolumn{3}{|c|}{$\begin{array}{l}\text { Augmentation de la durée } \\
\text { (p. 100) }\end{array}$} \\
\hline $\begin{array}{l}\text { Duodénum } \ldots \ldots \ldots \ldots \ldots \ldots \ldots \ldots \ldots \ldots \ldots \ldots \ldots \ldots \ldots \ldots \ldots \\
\text { Jéjunum } \ldots \ldots \ldots \ldots \ldots \ldots \ldots\end{array}$ & $\begin{array}{r}10,1 \\
7,4\end{array}$ & $\begin{array}{r}53,1 \\
8,1\end{array}$ & $\begin{array}{l}42,3 \\
73,4\end{array}$ & $\begin{array}{l}43,2 \\
48,6\end{array}$ \\
\hline $\begin{array}{l}\text { Temps de transif }(\min ) \ldots \ldots \ldots \ldots \ldots \ldots \ldots \ldots \\
\text { Débit jéjunal }(\mathrm{ml} / \mathrm{h}) \ldots \ldots \ldots \ldots \ldots \ldots \ldots \ldots \ldots \\
\text { Pouvoir de rétention d'eau }(\mathrm{ml} / \mathrm{g} \text { de matière } \\
\text { sèche) } \ldots \ldots \ldots \ldots \ldots \ldots \ldots \ldots \ldots \ldots \ldots \ldots \ldots \ldots\end{array}$ & 172 & $\begin{array}{r}12,0 \\
161 \\
5,5\end{array}$ & $\begin{array}{l}82,0 \\
55 \\
7,1\end{array}$ & $\begin{array}{l}14,0 \\
285 \\
22,8\end{array}$ \\
\hline
\end{tabular}

* Solka floc ; ** Normacol. 
La lignine est un polymère d'une centaine d'unités de phénylpropane ; elle forme de nombreuses liaisons, notamment avec les hémicelluloses dont elle diminue la digestibilité ; elle adsorbe en outre certains acides biliaires dont elle réduit la réabsorption.

L'intérêt des fibres alimentaires, dont la meilleure source est le son de blé, vient de ce qu'elles augmentent le volume et le poids des selles sans relation direcie avec leur pouvoir de rétention d'eau (tabl. 4). Le son est une arme efficace contre la constipation (ef la sédentarité), employée avec succès en élevage porcin (en particulier les truies en fin de gestation) et préconisée dès 1936 chez l'homme par Williams et Olmsted.

Intestin grêle. - L'effet des fibres a été longtemps nié, les modifications du temps de transit étant rapportées à un ralentissement de la vidange gastrique (McCance, Prior et Widdowson, 1953). Une autre explication est un effet négligeable sur l'intestin grêle par rapport à celui exercé sur le côlon. Or, sur le plan clinique, le syndrome côlon irritable est parfois associé à des troubles digestifs d'origine duodénale comme si une ration dont la teneur en fibres était incorrecte pouvait modifier à la fois l'activité du côlon et celle de l'intestin grêle.

Les carnivores, dont le régime est normalement pauvre en fibres, sont très sensibles à l'addition de fibres à leur ration : $50 \mathrm{~g}$ de son de blé, de cellulose ou de gommes augmente de 43 à 53 p. 100 la durée d'activité motrice continue postprandiale du duodénum (voir tabl. 4). L'effet est plus sélectif sur le jéjunum ; la cellulose purifiée et les gommes restent excito-motrices. La riposte motrice est fragmentée et les contractions groupées en épisodes moteurs de forte intensité entrecoupés de périodes silencieuses d'une à deux minutes pour la cellulose (fig. 9). Un tel profil moteur contraste avec celui

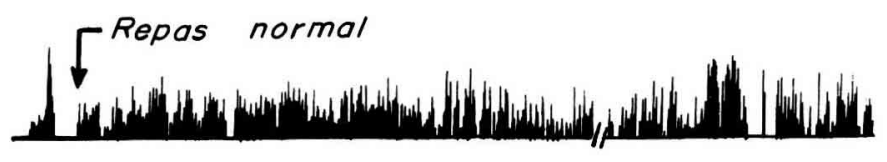

+ Son

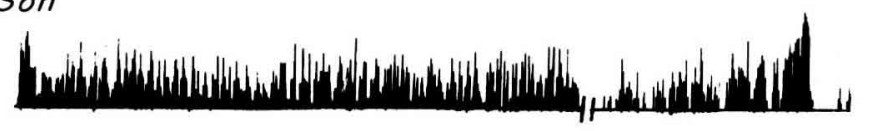

+ Cellulose

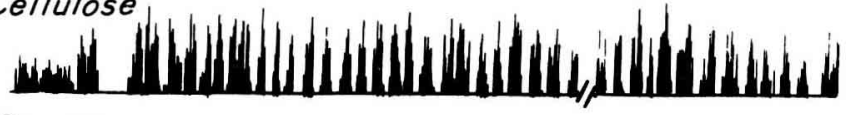

- Gormmes

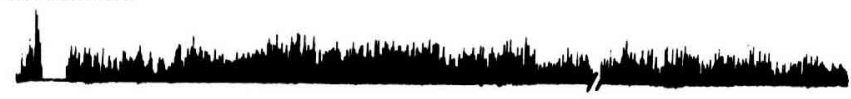

FIG. 9. - Profil moteur jéjunal ef fibres alimentaires chez le chien. L'addition à un repas standard de $50 \mathrm{~g}$ de son est caractérisée par une régularisation de l'activité motrice. Celle-ci est fragmentée pour la cellulose ef rigoureusement continue avec les gommes.

d'une activité continue mais de faible amplitude observée dans le cas des gommes (Bueno et al., 1981). Le temps de transit intestinal est ralenti de 7 à 10 fois dans le cas de la cellulose ; il l'est seulement de 20 à 30 p. 100 pour les gommes. Enfin, le son possède des propriétés intermédiaires, exception faite d'une activité excito-motrice qui s'exerce très sélectivement sur le duodénum (voir tabl. 4). La spécificité de cet effet des fibres est 
démontrée par l'obtention du même résultał après l'administration directe dans la lumière intestinale des fibres mélangées dans 200 ou $300 \mathrm{ml}$ d'eau.

Côlon. - L'effet des fibres sur la motricité colique a été chiffré chez l'homme pour lequel il existerait une relation logarithmique entre le poids quotidien des fèces et le temps de transit. La taille des fibres n'est pas sans importance. En effet, le poids des selles de 3 jours est accru lorsque la taille des particules excède le millimètre : $524 \mathrm{~g}$ au lieu de $394 \mathrm{~g}$ en régime normal pour des particules de son de 1 à $1,5 \mathrm{~mm}$ à la dose de 4 galettes de $4,5 \mathrm{~g}$ par jour ; le poids des selles n'est que de $384 \mathrm{~g}$ lorsque la faille des particules de son est inférieure au millimètre (Frexinos et Louis, 1978). Bien que la motricité du çôlon ne soit que légèrement modifiée, du moins d'après les critères de mesure utilisés, le temps de transit est, selon Brodibb ef al. (1980), réduit de moitié chez le macaque recevant $20 \mathrm{~g}$ de fibres par jour et le poids des selles est triplé (tabl. 5).

TABLEAU 5

Effef «fibres alimentaires» sur la motricité colique du macaque et du chien

\begin{tabular}{|c|c|c|c|c|}
\hline \multicolumn{5}{|c|}{ Macaque (Brodribb ef al., 1980) } \\
\hline $\begin{array}{c}\text { Teneur en fibres } \\
(\mathrm{g} / \mathrm{jour})\end{array}$ & $\begin{array}{l}\text { Poids des selles } \\
\text { (g) }\end{array}$ & \multicolumn{2}{|c|}{$\begin{array}{l}\text { Teneur en eau } \\
\text { (p. 100) }\end{array}$} & $\begin{array}{l}\text { Temps de transit } \\
\text { (h) }\end{array}$ \\
\hline $\begin{array}{r}0 \\
10 \\
20\end{array}$ & $\begin{array}{r}5,7 \\
9,6 \\
18,9\end{array}$ & & & $\begin{array}{l}82 \pm 8 \\
43 \pm 5 \\
38 \pm 3\end{array}$ \\
\hline \multicolumn{5}{|c|}{ Chien (Fioramonti ef al., 1980) } \\
\hline $\begin{array}{c}\text { Teneur en fibres } \\
\text { ( } g / \text { jour })\end{array}$ & $\begin{array}{l}\text { Poids des selles } \\
\text { (g) }\end{array}$ & $\begin{array}{l}\text { Teneur en eau } \\
(p .100)\end{array}$ & \begin{tabular}{l}
\multicolumn{2}{c}{ Motricité } \\
(p. 100) \\
$\left({ }^{1}\right)$
\end{tabular} & $\begin{array}{l}\text { Rapport } \\
\text { AP/AL ( }{ }^{(*)}\end{array}$ \\
\hline $\begin{array}{c}0 \\
4,4 \\
13,5\end{array}$ & $\begin{array}{r}30,3 \\
92,6 \\
183,4\end{array}$ & $\begin{array}{l}67,4 \\
79,0 \\
88,6\end{array}$ & $\begin{array}{l}51,1 \\
39,0 \\
51,7\end{array}$ & $\begin{array}{r}0,2 \\
2,6 \\
11,6\end{array}$ \\
\hline
\end{tabular}

(1) En p. 100 du temps d'enregistrement. ( ${ }^{2}$ ) Rapport d'activité propagée/activité localisée.

En réalité, les fibres ne constituent pas un « accélérateur » mais plutôt un « régulateur » du transit en ce sens que l'addition de son de blé au régime permet de rétablir un temps de transit normal chez des sujets qui présentent un temps de transit long (supérieur à $60 \mathrm{~h}$ ) ou court (inférieur à $36 \mathrm{~h}$ ). Ainsi, l'addition de son est sans effet chez le porc dont le temps de transit est normal (Canguilhem et Labie, 1977). Enfin, l'action d'un type de fibres évaluée par le poids des selles émises est autant plus importante que le pouvoir de rétention d'eau est faible (Stephen et Cummings, 1979). L'explication de ce résultat en apparence paradoxal est simple : l'attaque bactérienne plutôt que l'effet moteur va de pair avec le pouvoir hydrophile des fibres ; les fibres « ne retenant pas l'eau » sont fortement excito-motrices et augmentent le poids des selles émises.

Le mécanisme qui sous-tend l'accélération du transit en présence de fibres a été examiné chez le porc. L'addition de son de blé grossier contenant 45 p. 100 d'hémicelluloses, 23 p. 100 de cellulose ef 9 p. 100 de lignine à un régime lait ( 3 p. 100 d'hémicellulose) pour lequel le temps de transit est voisin de $120 \mathrm{~h}$, ramène le transit à $66 \mathrm{~h}$ par le seul renforcement de l'activité propagée. Le nombre quotidien de salves de 
contractions passe de 510 à 1066 , la durée de l'activité localisée étant inchangée (Fioramonti et Bueno, 1980). Chez le chien de $14-18 \mathrm{~kg}$ qui reçoit $3 \mathrm{à} 400 \mathrm{~g}$ de viande crue, l'activité contractile du côlon occupe environ 50 p. 100 du temps d'enregistrement pour $30 \mathrm{~g}$ de fèces pauvres en eau (voir tabl. 5). L'activité motrice occupe également 50 p. 100 du temps d'enregistrement chez un chien acceptant de consommer $500 \mathrm{~g}$ d'un régime riche en céréales (13,5 p. 100 de fibres), mais le poids des fèces riches en eau est de $180 \mathrm{~g}$. Le rapport des deux types d'activité est considérablement modifié pour ces deux sifuations extrêmes, au demeurant entrecoupées d'épisodes de constipation avec la viande et d'état diarrhéique avec les céréales.

L'activité propagée du côlon est quasi inexistante chez le carnivore ; elle esł prépondérante chez le végétarien. Inversement, l'activité localisée est importante pour les régimes à faible taux de résidus (fig. 10).

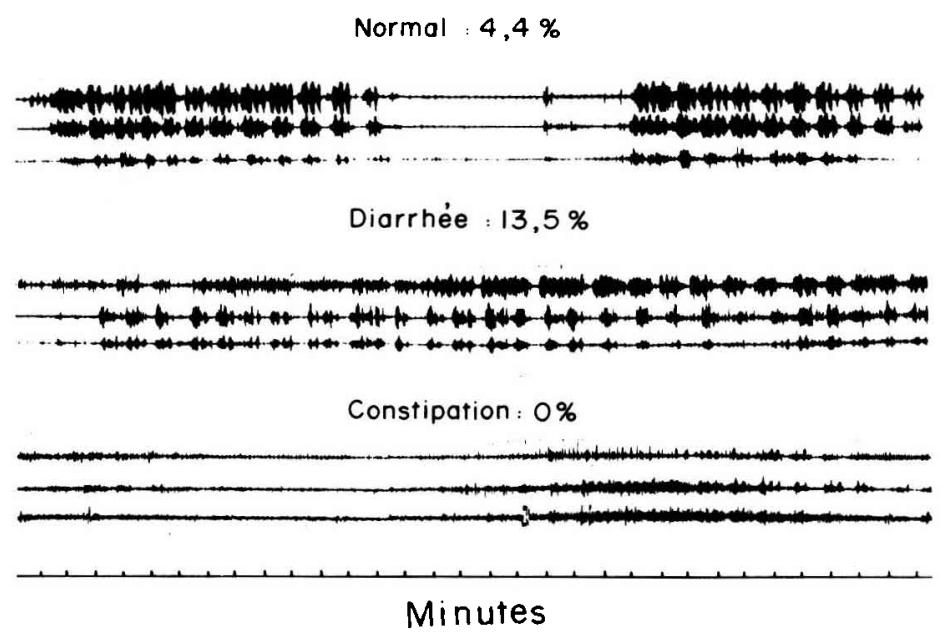

FIG. 10. - Motricité colique ef fibres alimentaires chez le chien. Les périodes d'activité localisée et propagée occupent environ 30 p. 100 du temps d'enregistrement pour un régime à 4,4 p. 100 de fibres. L'activité est localisée dans le cas où la ration ne contient pas de fibres (constipation) ef elle est propagée continue pour un régime à 13,5 p. 100 de fibres (diarrhée).

Le mécanisme probable de l'effet «fibres» semble d'ordre physique chez le chien où la distension d'un viscère creux est maximale dès que le régime n'est pas celui d'un carnivore, sensu stricto (Bueno ef al., 1981). En revanche, chez le lapin, le porc ef l'homme, où les produits de la dégradation bactérienne des fibres sont quantitativement importants, une action pharmacodynamique ne peut être exclue. Ainsi, chez le lapin, le remplacement de la cellulose par de la vermiculite $(10$ p. 100$)$ entraîne une diminution du gain de poids (18 g/jour au lieu de 24) selon Colin ef al. (1976) ; or, cette perte de poids est supérieure à celle que compenserait l'énergie apportée par les acides gras volatils de 10 p. 100 de cellulose.

\subsection{Effets métaboliques.}

Un régime riche en fibres favorise l'élimination des graisses, du calcium, du phosphore et des acides biliaires. Le fait a été illustré en médecine vétérinaire par la mala- 
die du son ou ostéofibrose du cheval. La déformation des os nasaux avec profil busqué du chanfrein est le premier signe de la maladie dont étaient surtout atteints les chevaux de meunier.

Les flèches de l'hyperglycémie provoquée ef les oscillations de l'insulinémie sont atténuées chez l'homme dans le cas d'un régime riche en pectines (Wolever et al., 1979). Cependant, cette action métabolique des fibres sur les différents constituants du sang est discrète. Selon Collings ef al. (1979), le remplacement chez le porc d'une partie du maïs de la ration par 10 à 30 p. 100 de remoulures de blé, ce qui augmente de 0,3 à 1,7 p. 100 la teneur en fibres du régime, a pour effet seulement de diminuer de 10 à 20 p. 100 la glycémie et l'absorption phospho-calcique.

Une action plus importante des fibres serait liée aux acides gras volatils qui, par un effef cathartique, augmenteraient le volume des selles (Cummings ef al., 1976). L'hypothèse d'un effet de fype osmotique plutôt qu'une action pharmacodynamique excitomotrice est plausible car les acides gras volatils, loin d'être excito-moteurs vis-à-vis du côlon, sont plutôt inhibiteurs ; ils le sont toujours pour le cæcum dont l'atonie ainsi créée serait un facteur favorisant son déplacement chez les ruminants (Svendsen, 1972).

L'action des acides gras volatils par le biais de modifications de la pression osmotique du contenu colique a été démontrée chez le cheval ; l'inhibition de l'absorption hydroélectrolytique en présence d'un excès d'acides gras volatils expliquerait l'élimination épisodique de selles trop liquides (Argenzio, Miller et von Engelhardt, 1975) ; mais surtout, il existe une relation directe entre la concentration en acides gras volatils ef la pression osmotique des fèces chez les chevaux qui souffrent d'épisodes diarrhéiques, quelle que soit leur étiologie (Merritt et Smith, 1980).

Un as pect non évoqué ici est la production des gaz et leur interférence sur la motricité. Des preuves existent d'un transit très rapide et du signe pronostique très favorable constitué par leur évacuation dans le cas d'une occlusion intestinale transitoire.

\subsection{Adaptations anatomo-fonctionnelles.}

La diverticulose colique, qui concerne surtout le côlon sigmoïde, se traduit par la présence de hernies de la paroi entre les faisceaux de la couche circulaire au point de passage des vaisseaux sanguins. Elle serait consécutive à une pression endoluminale trop élevée, elle-même liée à une aciivité motrice trop importante en l'absence de fibres. Cette hypothèse fait de la maladie diverticulaire une conséquence d'une alimentation pauvre en fibres, facilitant l'hypertonie du côlon proximal ; elle reste très discutée pour plusieurs raisons :

- la carence en fibres modifie moins la durée d'activité motrice du côlon que le type d'activité ;

- l'excès de fibres, et non la carence, entraîne l'hypertrophie de la paroi, support d'une forte activité motrice ;

- une teneur élevée ou faible en fibres de la ration s'accompagne de modifications motrices au moment du changement de régime; mais à terme, la réponse à un régime stable même déséquilibré est d'ordre morphologique.

Chez le rat, une même quantité hebdomadaire d'aliments ingérés en 4 jours ne modifie pas quantitativement l'activité contractile de l'intestin (Ruckebusch et Ferré, 1972). Au bout de 3 à 4 semaines, c'est la capacité digestive qui est accrue grâce à une 
TABLEAU 6

Adaptations morphologiques du tractus digestif (croissance pondérale normale)

Rat : Jeône intermittent de 3 j par semaine pendant 5 mois (Ruckebusch et Ferré, 1972)

\begin{tabular}{|c|c|c|c|c|}
\hline & \multicolumn{2}{|c|}{ Témoins } & \multicolumn{2}{|c|}{ Jeûne } \\
\hline & Estomac & Infestin & Estomac & Intestin \\
\hline $\begin{array}{l}\text { Epaisseur totale }\left(\mathrm{mm} \times 1^{-3}\right) \quad \ldots \ldots \ldots \ldots \ldots \ldots \\
\text { Poids (en p. 100) } \ldots \ldots \ldots \ldots \ldots \ldots \ldots \ldots \ldots\end{array}$ & $\begin{array}{l}417 \\
0,58\end{array}$ & $\begin{array}{l}107 \\
2,34\end{array}$ & $\begin{array}{l}410 \\
0,62\end{array}$ & $\begin{array}{r}157 \\
2,95\end{array}$ \\
\hline \multicolumn{5}{|c|}{ Rat : Régime sans et avec fibres (20 p. 100) (Ward et al., 1979) } \\
\hline & & Sans fibre & \multicolumn{2}{|c|}{ Avec fibres } \\
\hline $\begin{array}{l}\text { Poids du côlon (en } \mathrm{g}) \\
\text { Epaisseur }\left(\mathrm{mm} \times 10^{-1}\right)\end{array}$ & & 2143 & \multicolumn{2}{|c|}{3328} \\
\hline $\begin{array}{l}\text { c. circulaire } \ldots \ldots \ldots \ldots \ldots \ldots \ldots \ldots \ldots \ldots \ldots \ldots \ldots \ldots \ldots \ldots \ldots \ldots \\
\text { c. longitudinale } \ldots \ldots \ldots \ldots \ldots \ldots \ldots\end{array}$ & $\cdots$ & $\begin{array}{l}1,53 \\
0,30\end{array}$ & \multicolumn{2}{|r|}{$\begin{array}{l}2,39 \\
0,49\end{array}$} \\
\hline
\end{tabular}

Lapin : Régime isocalorique, isoazoté et isocellulosique (Candau ef al., 1978)

\begin{tabular}{|c|c|c|}
\hline & $\begin{array}{l}\text { Luzerne }\left({ }^{(1)}\right) \\
\text { Estomac Cacum }\end{array}$ & $\begin{array}{l}\left.\text { Pulpe ( }{ }^{2}\right) \\
\text { Estomac Cæcum }\end{array}$ \\
\hline $\begin{array}{l}\text { Volume }\left(\mathrm{cm}^{3}\right) \\
\text { Vitesse d'ingestion }(\mathrm{g} / \mathrm{min}) \\
\text { Vin }\end{array}$ & $\begin{array}{l}40 \\
1,19 \pm 0,11\end{array}$ & $\begin{array}{l}80 \\
0,42\end{array} \quad \begin{array}{l}400 \\
0,07\end{array}$ \\
\hline $\begin{array}{l}\text { Utilisation digestive } \\
\quad \text { Hémicelluloses } \ldots \ldots \ldots \ldots \ldots \ldots \ldots \ldots \ldots \ldots \ldots \ldots \ldots \ldots \\
\quad \text { Cellulose vraie } \ldots \ldots \ldots \ldots \ldots \ldots \ldots \ldots \ldots \\
\text { Contractions cæcales par g d'aliment } \ldots \ldots \ldots \ldots \ldots\end{array}$ & $\begin{array}{l}52,1 \pm 4,2 \\
22,0 \pm 4,9 \\
19,6 \pm 1,9\end{array}$ & $\begin{array}{l}76,5 \pm 5,7 \\
50,2 \pm 8,8 \\
20,4 \pm 2,2\end{array}$ \\
\hline
\end{tabular}
5 p. 100.

(1) «Crude fiber " : 15,6 p. 100 ; cellulose vraie : 14,8 p. 100 ; hémicellulose : 18,5 p. 100 ; lignine :

$\left({ }^{2}\right)$ "Crude fiber" : 14,9 p. 100 ; cellulose vraie : 15,1 p. 100 ; hémicellulose : 23,5 p. 100 ; lignine : 3,5 p. 100.

hypertrophie de l'estomac et de l'intestin grêle (tabl. 6). L'adaptation morphologique et non fonctionnelle se retrouve également chez le rat recevant un régime riche en fibres (20 p. 100 de la ration) capable de tripler le poids des fèces (18 $\mathrm{g}$ au lieu de 6). L'épaisseur de la couche de fibres musculaires circulaires de la paroi colique est alors accrue de 40 p. 100 . Selon Ward, Lewin ef Clark (1979), ce résultat va à l'encontre d'une relation diverticulose-manque de fibres; la maladie diverticulaire serait davantage liée à des transitions non ménagées pour des régimes dont la teneur en fibres est variable qu'à un régime « déséquilibré » de façon constante. Le même type d'adaptation gastro-colique a été observé chez le porc recevant de la luzerne (Bohman, Kidwell et McCormick, 1953).

Enfin, chez le lapin, l'adaptation peut être suffisante pour ne pas gêner la croissance. Ainsi, le poids et le volume du cæcum sont accrus chez le lapin qui reçoit, au lieu de luzerne déshydratée, un aliment à base de pulpe de betteraves, riches en hémicelluloses. Le volume de l'estomac, celui du cæcum et du côlon dont les valeurs de référence sont connues (Lebas et Laplace, 1972) sont augmentés de 5 à 10 p. 100 ; la teneur en matière sèche du contenu cæcal « pulpe » est de 21 p. 100 au lieu de 26 p. 100 pour le contenu « luzerne » et la quantité de contenu cæcal est de $180 \mathrm{~g}$ au lieu de $100 \mathrm{~g}$ à l'âge de 3 mois (Candau, Bertrand et Fioramonti, 1978). La présence d'hémicelluloses ralen- 
tit vraisemblablement ici la vidange gastrique mais pas au point de modifier l'évolution de la courbe pondérale qui reste comparable de même que l'activité motrice intestinale globale.

\section{Conclusions.}

Non seulement les propriétés sécrétrices mais aussi à court terme l'activité motrice du tube digestif et à long terme sa capacité sont modifiées par le contenu délivré par le réservoir gastrique.

- Tous les constituants alimentaires sont susceptibles d'intervenir. Les uns sont absorbés au niveau de l'intestin grêle et c'est l'absorption elle-même qui déclenche soit une action excitomotrice et dynamogène soit une inhibition transitoire de la motricité. Les autres, qualifiés de fibres, ne sont que partiellement dégradés en région distale et leur effet sur le transit est essentiellement celui d'une modification du rapport activité localisée/activité propagée du côlon.

- Les effets des constituants ne sont pas nécessairement similaires sur les deux parties de l'intestin. Ainsi les matières grasses, inhibitrices en zone duodéno-jéjunale, sont excito-motrices en zone colique. De même, la cellulose réduit le débit jéjunal au tiers de sa valeur alors qu'elle double celui du côlon. Enfin, la structure physique et la composition chimique des aliments peuvent être des facteurs de modification des trois éléments déterminants : le transit digestif, la capacité du contenant et la durée ou le type d'activité motrice dont il est le siège.

- Les réponses du tube digestif à son contenu sont donc rapides pour les modifications motrices et lentes pour les variations volumétriques. Le fait que le profil moteur de base soit désormais bien individualisé pour l'intestin grêle et pour le côlon, et cela quelle que soit l'espèce animale envisagée, facilite l'étude et la compréhension du rôle des facteurs alimentaires dans la motricité.

Journées Digestion-Absorption de l'Association française de Nutrition,

Toulouse-Auzeville, 20-21 novembre 1980.

\section{Références}

ARGENZIO R. A., MILLER N., VON ENGELHARDT W., 1975. Effect of volatile fatty acids on water and ion absorption from the goat colon. Am. J. Physiol., 229, 997-1002.

AUFFRAY P., MARTINET J., RÉRAT A., 1967. Quelques aspects du transit gastro-intestinal chez le porc. Ann Biol. anim. Bioch. Biophys., 7, 261-279.

BOHMAN V. R., KIDWELL J. F., MCCORMICK J. N., 1953. High levels of alfalfa in the rations of growing fattening swine. J. anim. Sci., 12, 876-882.

BRODRIBB J., CONDON R. E., COWLES V., DECOSSE J. J., 1980. Influence of dietary fiber on transit time, fecal composition, and myoelectrical activity of the primate right colon. Dig. Dis. Sci., 25, 260-266.

BUENO L., 1977. Complexe myoélectrique de l'intestin grêle et variations de l'apport alimentaire chez le mouton. C. R. Soc. Biol., 171, 959-964.

BUENO L., FIORAMONTI J., RUCKEBUSCH Y., 1975. Rate of flow of digesta and electrical activity of the small intestine in dogs and sheep. J. Physiol. (Lond.), 249, 69-85.

BUENO L., FIORAMONTI J., RUCKEBUSCH Y., FREXINOS J., COULOM P., 1980. Evaluation of colonic myoelectrical activity in health and functional disorders. Gut, 21, 480-485. 
BUENO L., PRADDAUDE F., FIORAMONTI J., RUCKEBUSCH Y., 1981. Effect of dietary fiber on gastro-intestinal motility and jejunal transit time in dogs. Gastroenterology, 80, 701-707.

BUENO L., RUCKEBUSCH M., 1976. Insulin and jejunal electrical activity in dogs and sheep. Am. J. Physiol., 230, 1538-1544.

BUENO L., RUCKEBUSCH Y., 1978. Migrating myoelectric complexes : disruption, enhancement and disorganization, 89-91. In DUTHIE M. L. Gastrointestinal motility in health and disease. MTP Press Ltd Lancaster.

BURKITT D. P., 1976. A deficiency of dietary fiber may one cause of certain colonic and venous disorders. Am. J. Dig. Dis., 21, 104-108.

CANDAU M., BERTRAND B., FIORAMONTI J., 1978. Variations de la digestibilité des constituants de la ration chez le lapin. C. $R$. Soc. Biol., 172, 554-560.

CANGUILHEM R., LABIE C., 1977. Variation de la durée du transit intestinal chez le porc selon la leneur de la ration en cellulose. Revue Méd. vét., 128, 1669-1681.

CODE C. F., MARLETT J. A., 1975. The interdigestive myoelectric complex of the stomach and small bowel of dogs. J. Physiol. (Lond.), 246, 289-309.

COLIN M., MAIRE C., VAISSAIRE J., RENAUL T., 1976. Ełude expérimentale du remplacement dans les aliments pour lapin de la cellulose par des lests minéraux. Rec. Méd. vét., 152, 457-465.

COLLINGS G. F., ERICKSON J. P., YOKOYAMA M. T., MILLER E. R., 1979. Effect of wheat middlings on fiber digestibility, serum cholesterol and glucose and fecal bile acids in pigs. J. anim. Sci., 49, 528-534.

CONNELL A. M., LOGAN C. J. H., 1967. The role of gastrin in gastro-ileo-colic responses. Am. J. dig. Dis., 12, 277-284.

CORRING T., MOUROT J., CHARLES M., LEGER C., 1979. Dérivation de la sécrétion biliaire et activités enzymatiques dans le tissu pancréatique chez le porc. Ann. Biol. anim. Bioch. Biophys., 19, 833-836.

CUMMINGS J. H., HILL M. J., JENKINS D. J. A., PEARSON J. R., WIGGINS H. S., 1976. Changes in fecal composition and colonic function due to cereal fiber. Am. J. clin. Nutr., 29, 1468-1473.

DARDILLAT C., 1977. Analyse électromyographique et débitmétrique du transit alimentaire chez le veau nouveau-né. J. Physiol. (Paris), 73, 925-944.

DARDILLAT C., MARRERO E., 1977. Ełude de l'électromyogramme global chronique de la paroi intestinale de veau préruminant : migration des phases d'activité régulière et relation avec le transif. Ann. Biol. anim. Bioch. Biophys., 17, 523-530.

DE WEVER I., EECKHOUT C., VANTRAPPEN G., HELLEMANS J., 1978. Disruptive effect of test meals on interdigestive motor complex in dogs. Am. J. Physiol., 235, E661-E665.

ELLIOTT T. R., BARCLAY-SMITH E., 1904. Antiperistalsis and other muscular activities of the colon. J. Physiol. (Lond.), 31, 272-283.

FIORAMONTI J., BUENO L., 1980. Motor activity in the large intestine of the pig related to dietary fibre and retention time. Br. J. Nutr., 43, 155-162.

FIORAMONTI J., BUENO L., RUCKEBUSCH Y., 1980. Dualité de l'activité myoélectrique du côlon chez l'animal. Reprod. Nutr. Dévelop., 20, 1203-1208.

FIORAMONTI J., GARCIA-VILLAR R., BUENO L., RUCKEBUSCH Y., 1980. Colonic myoelectrical activity and propulsion in dog. Dig. Dis. Sci., 25, 641-646.

FIORAMONTI J., RUCKEBUSCH Y., 1976. La motricité cæcale chez le lapin. 3. - Dualité de l'excrétion fécale. Ann. Rech. vét., 7, 281-295.

FIORAMONTI J., RUCKEBUSCH Y., 1979. Diet and caecal motility in sheep. Ann. Rech. vét., 10, $593-$ 599.

FREXINOS J., 1979. Intérêt des fibres alimentaires en pathologie digestive. Ann. Nutr. Alim., $33,199$. 210.

FREXINOS J., LOUIS A., 1978. Effet sur le poids des selles de trois produits contenant des fibres alimentaires. Gastroenterol. clin. biol., 2, 1055-1056.

GOODALL E. D., KAY R. N. B., 1965. Digestion and absorption in the large intestine of the sheep. J. Physiol. (Lond.), 176, 12-33.

HECKER J. F., GROVUM W. L., 1975. Rates of passage of digesta and water absorption along the large intestines of sheep, cows and pigs. Aust. J. biol. Sci., 28, 161-167.

HOLDSTOCK D. J., MISIEWICZ J. J., 1970. Factors controlling colonic motility : colonic pressures and transit after meals in patients with total gastrectomy, pernicious anaemia or duodenal ulcer. Gut, 11, 100-110. 
KASS M. L., VAN SOEST P. J., POND W. G., 1980. Utilisation of dietary fiber from alfalfa by growing swine. II. VFA acid concentration and disappearance from the G.I. tract. J. Nutr., 50, 192-197.

KIDDER D. E., MANNERS M. J., 1978. Digestion in the pig. Ed. Scientechnica, Bristol. 201 pP.

KIRWAN W. O., SMITH A. N., 1976. Posłprandial changes in colonic motility related to serum gastrin levels. Scand. J. Gastroenterol., 11, 145-149.

LAPLACE J. P., 1978. Complexes myoélectriques de l'intestin grêle, motricité cæcale et diarrhée. Ann. Zootech., 27, 495-517.

LAPLACE J. P., 1980. Stomach and small intestine motility : electromyography in nutritional studies, 24-27. In LOW A. G., PARTRIDGE I. G., Current concepts of digestion and absorption in pigs, NIRD Press, Shinfield.

LEBAS F., LAPLACE J. P., 1972. Mensurations viscérales chez le lapin. 1. Croissance du foie, des rein et des divers segments intestinaux entre 3 et 11 semaines. Ann. Zootech., 21, 37-47 et 505-524.

MCCANCE R. A., PRIOR K. M., WIDDOWSON E. M., 1953. A radiological study of the rate of passage of brown and white bread through the digestive tract of man. Brit. J. Nutr., 7, 98-104.

MERRITT A. M., SMITH D. A., 1980. Osmolarity and volatile fatty acid content of feces from horses with chronic diarrhea. Am. J. vet. Res., 41, 928-931.

RAYNER V., WENHAM G., WHITE F., RHIND S. M., BRUCE J. B., 1980. Digesta transit by X-ray screening, glucose absorption, insulin secretion, and the MMC in the pig, 253-260. In CHRISTENSEN J. Gastrointestinal motility. Raven Press, New York.

RÉRAT A., 1978. Digestive and absorption of carbohydrates and nitrogenous matters in the hindgut of the omnivorous non ruminant animal. J. anim. Sci., 46, 1808-1897.

RÉRAT A., LOUGNON J., 1963. Ełudes sur le transit digestif chez le porc. Annls. Biol. anim. Bioch. Biophys., 3, 21-29.

RINAUDO M., 1980. Structure et caractérisation des principaux constituants des fibres alimentaires. Ann. Nutr. Alim., 34, 57-76.

RUCKEBUSCH Y., 1981. Motor functions of the intestine. Adv. vet. Sci. comp. Med., 25, 345-369.

RUCKEBUSCH Y., BUENO L., 1976. The effect of feeding on the motility of the small intestine in the pig. Br. J. Nutr., 35, 397-405.

RUCKEBUSCH Y., BUENO L., 1977. Migrating myoelectrical complex of the small intestine. An intrinsic activity mediated by the vagus. Gastroenterology, 73, 1309-1314.

RUCKEBUSCH M., FERRÉ J. P., 1972. Modifications digestives liées au jeône intermittent chez le rat. C. R. SoC. Biol., 166, 1552-1567.

RUCKEBUSCH M., FIORAMONTI J., 1975. Electrical spiking activity and propulsion in small intestine in fed and fasted rats. Gastroenterology, 68, 1500-1508.

RUCKEBUSCH Y., FIORAMONTI J., 1980a. Colonic myoelectrical spiking activity : major patterns and significance in six different species. Zbl. Vef. Med., 27, 1-8.

RUCKEBUSCH Y., FIORAMONTI J., 1980b. Motor profile of the ruminant colon : hard vs soft faeces. Experientia, 36, 1184-1185.

SCHANG J. C., SAVA P., ANGEL F., LAMBERT A., GRENIER J. F., 1978. Specific effects of different food components on intestinal motility. Electromyographic study in dogs. Eur. surg. Res., 10, 425-432.

SCHANG J. C., SAVA P., ANGEL F., BOUCHET P., GRENIER J. F., 1980. Fat-induced postprandial intestinal motility : relation between its duration and lipidic ileal output in the dogs, 353-358. In CHRISTENSEN J., Gastrointestinal motility, Raven Press, New York.

SNAPE W. J., MATARAZZO S. A., COHEN S., 1978. Effect of eating and gastrointestinal hormones on human colonic myoelectrical and motor activity. Gastroenterology, 75, 373-378.

SNAPE W. J., WRIGHT S. H., BATTLE W. M., COHEN S., 1979. The gastrocolic response : evidence for a neural mechanism. Gastroenterology, 77, 1235-1240.

SPILLER G. A., SHIPLEY E. A., BLAKE J. A., 1978. Recent progress in dietary fiber (plantix) in human nutrition. Food Sci. Nutr., 10, 31-90.

STEPHEN A. M., CUMMINGS J. H., 1979. Water-holding by dietary fibre in vitro and its relationship to faecal output in man. Gut, 20, 722-729.

SVENDSEN P., 1972. Inhibition of cecal motility in sheep by volatile fatty acids. Nord. vet. Med., 24, 393-396.

TANSY M. F., KENDALL F. M., 1973. Experimental and clinical aspects of gastrocolic reflexes. Am. J. Dig. Dis., 18, 521-531. 
TEMPLETON R. D., LAWSON H., 1931. Studies in the motor activity of the large intestine. I. Normal motility in the dog, recorded by the tandem balloon method. Am. J. Physiol., 96, 667-676.

THIVEND P., 1979. La digestion des glucides chez le veau non sevré. Ann. Nutr. Alim., 33, 233-245. TROWEL H., 1976. Definition of dietary fiber and hypothesis that is a protective factor in certain diseases. Am. J. clin. Nutr., 29, 417-427.

WALKER A. R. P., 1947. The effect of recent changes of food habits on bowel motility. S. Afr. med. J., 21, 590-593.

WARD M. W., LEWIN M. R., CLARK C. G., 1979. The influence of dietary fibre on colonic muscle. Eur. surg. Res., 11, 360-365.

WEISBRODT N. W., 1981. Patterns of infestinal motility. Ann. Rev. Physiol., 42, 21-31.

WILLIAMS R. D., OLMSTED W. H., 1936. The effect of cellulose, hemicellulose and lignin on the weight of the stool. A contribution to the study of laxation in man. J. Nutr., 11, 433-449.

WOLEVER T. M. S., JENKINS D. J. A., NINEHAM R., ALBERTI K. G. M. M., 1979. Guar gum and reduction of postprandial glycaemia : effect of incorporation into solid food, liquid food, and both. Br. J. Nutr., 41, 505-510.

WRIGHT S. H., SNAPE W. J., BATTLE W., COHEN S., LONDON R. L., 1980. Effect of dietary components on gastrocolonic response. Am. J. Physiol., 238, G228-G232. 